\title{
Cell budding from pre-invasive tumors: Intrinsic precursor of invasive breast lesions?
}

\author{
JIN-HONG GAI ${ }^{1,2}$, PENG-TAO GONG ${ }^{2}$, JIAN-HUA LI $^{2}$, YAN-GAO MAN ${ }^{5}$, \\ JIN-SONG NI ${ }^{3}$, HONGXI MA ${ }^{3}$, FEN-YUN HAO ${ }^{4}$, XI-CHEN ZHANG ${ }^{2}$ and YING LIU ${ }^{1}$
}

\author{
${ }^{1}$ Faculty of Veterinary Medicine, Gansu Agricultural University, Lanzhou, Gansu 730070; ${ }^{2}$ College of Animal Science and \\ Veterinary Medicine, and ${ }^{3}$ Department of Pathologic Anatomy, School of Basic Medical Sciences, Jilin University, \\ Changchun, Jilin; ${ }^{4}$ Department of Pathology, Weifang People's Hospital, Weifang, Shandong 261061, P.R. China; \\ ${ }^{5}$ Department of Gynecologic and Breast Pathology, Armed Forces Institute of Pathology and \\ American Registry of Pathology, Washington, DC 20306-6000, USA
}

Received February 4, 2011; Accepted April 1, 2011

DOI: 10.3892/etm.2011.251

\begin{abstract}
Our previous studies showed that in patients with ductal carcinoma in situ (DCIS) of the breast, the tumor cells that overlie focal myoepithelial cell layer disruptions (FMCLDs) are generally arranged as finger-like projections that bud into the stroma. These budding cells have significantly more genetic instability and invasion-related gene expression, and less estrogen receptor (ER) expression, than their epithelial cell counterparts. This study aimed to assess these cells for potential molecular markers that are uniquely associated with cell adhesion and motility. Seventeen ER-positive DCIS cases were screened by immunostaining for ER, and 7 cases which harbored FMCLD lesions were used to examine the expression of the potential markers. Two cases with both DCIS and invasive lesions were selected for comparing the differences in molecular expression between these lesion types. The results showed that expression levels of talin, E-cadherin and focal adhesion kinase (FAK) in tumor cells overlying FMCLDs were higher than those within the corresponding duct. Integrin $\beta 1$ staining was detected only in a small number of the tumor cells overlying the FMCLDs. Vinculin staining was weak $(18 \%)$ or not detected $(82 \%)$, and no expression was found in the tumor cells within the corresponding duct or in the pure isolated DCIS. By contrast, the expression levels of talin, vinculin and integrin $\beta 1$ in the invasive tumors were distinctly higher than those in DCIS, and the expression of FAK and E-cadherin was lower. Using electron microscopy, we found that the tight junctions between tumor
\end{abstract}

Correspondence to: Dr Xi-Chen Zhang, College of Animal Science and Veterinary Medicine, Jilin University, Changchun, P.R. China

E-mail: xczhang@jlu.edu.cn

Key words: ductal carcinoma in situ, talin, vinculin, focal adhesion kinase, integrin $\beta 1$ cells overlying the FMCLDs were reduced compared to the adjacent tumor cells in the lumen. These results indicate that the tumor cells overlying FMCLDs are likely to represent the specific precursors of invasive breast lesions. Our findings may also facilitate the identification of specific targets for further molecular profiling, which will more completely characterize this important cell population.

\section{Introduction}

It is a commonly held belief that ductal carcinoma in situ (DCIS) is the precursor of invasive breast lesions (1-3). The epithelium that lines the mammary ducts of the normal human breast and encloses in situ breast carcinomas, is physically separated from the stroma by both a myoepithelial (ME) cell layer and basement membrane (4-8). Most tumor epithelial cells must pass from the lumen of the duct through the ME cell layer, which makes the physical disruption of the myoepithelium a prerequisite for breast tumor invasion (9). Our previous studies noted the correlation between alterations of the myoepithelium and that of adjacent epithelial cells. Approximately $15 \%$ of pre-invasive breast tumors have been found to be associated with focal myoepithelial cell layer disruptions (FMCLDs) (9-13). At or near these breaks in the myoepithelium, tumor cells are generally arranged as fingerlike projections, budding from these disruptions. The cells overlying these disruptions have significantly less estrogen receptor (ER) expression, and also show greater proliferation, genetic instability and invasion-related gene expression than their morphologically similar counterparts within the same duct (9-13). It is well known that cell adhesion and motilityrelated molecules may play a critical role in the forward progression of breast carcinoma. These molecules include $\alpha$-smooth muscle actin (SMA), epithelial (E)-cadherin, integrins, talin, focal adhesion kinase (FAK) and vinculin (14-16).

The present study attempted to elucidate the histological and ultrastructural features of tumor cells that overlie FMCLDs, with respect to the uniqueness of vinculin, talin, E-cadherin, integrins and FAK, and to determine whether 
they may be qualified as biomarkers for early detection of breast tumor invasion.

\section{Materials and methods}

Breast cancer samples. Formalin-fixed paraffin-embedded (FFPE) blocks of breast cancer tissue were obtained from Weifang People's Hospital, First Hospital of Jilin University and China-Japan Union Hospital of Jilin University. Consecutive sections $(4-5 \mu \mathrm{m})$ were cut and placed on positively charged microscope slides. All cases were DCIS as determined by $\mathrm{H} \& \mathrm{E}$ staining. We screened 17 ER-positive cases to assess the molecules related to cell adhesion and motility, and selected 2 cases with combined DCIS and invasive lesions to compare to those of pure DCIS. The characteristics of the patients are listed in Table I.

Reagents and procedures. Antibodies reported to be associated with motility and adhesion of epithelial cells were selected for this study (Table II). These included antibodies against $\alpha$-SMA, FAK, integrin $\beta 1$, talin, vinculin and E-cadherin. ER antibody was used to screen the ER-positive cases and assess the ER-negative cell clusters overlying FMCLDs. $\alpha$-SMA antibody was used to examine the disruption of the ME cell layer. The immunohistochemical staining kit was purchased from Maxin Biocompany (Fuzhou, China). The double immunohistochemical staining kit was purchased from Zhongshan Biocompany (Beijing, China).

Immunohistochemical staining. The protocol for immunohistochemical staining has been previously published (17). All immunochemical staining section images were digitized using a TCA-9.0C CMOS microscope camera (Tucsen Imaging Technology) and Image-Pro plus software (Media Cybernetics, Silver Spring, MD, USA). The statistical analysis of the integrated optical density (IOD) was performed using Minitab and GraphPad software.

Evaluation of immunostaining. All slides stained using immunohistochemistry were double read by two independent observers and scored as negative $(-)$, weak $(+)$, moderate $(++)$ or strong $(+++)$.

FFPE tissue blocks transformed to ultra-thin sections. Using the H\&E staining results to determine the location
Table I. Patient characteristics.

\begin{tabular}{cccc}
\hline Case & Age (years) & Tumor type & ER \\
\hline 1 & 58 & DCIS, grade I & ++ \\
2 & 60 & DCIS, grade II & +++ \\
3 & 44 & DCIS, grade II & ++ \\
4 & 57 & DCIS, grade I & ++ \\
5 & 43 & DCIS, grade I & +++ \\
6 & 37 & DCIS, grade II & ++ \\
7 & 62 & DCIS, grade I & +++ \\
8 & 51 & DCIS, grade II & +++ \\
9 & 47 & DCIS, grade III & + \\
10 & 53 & DCIS, grade I & +++ \\
11 & 48 & DCIS, grade II & ++ \\
12 & 53 & DCIS, grade II & ++ \\
13 & 45 & DCIS, grade II & +++ \\
14 & 42 & DCIS, grade I & +++ \\
15 & 48 & DCIS, grade II & ++ \\
16 & 53 & DCIS, grade II & + \\
17 & 49 & DCIS, grade I & +++ \\
18 & 52 & DCIS and invasive tumor & + \\
19 & 47 & DCIS and invasive tumor & - \\
\hline & & & + \\
\hline & & &
\end{tabular}

ER, estrogen receptor; DCIS, ductal carcinoma in situ.

of interest in the FFPE tissue blocks, each was trimmed to approximately $2 \times 2 \times 2 \mathrm{~mm}^{3}$. The small block was packed with lens tissue, and deparaffinized in xylene for $40 \mathrm{~h}$. The block was then passed through a graded acetone series and rinsed in $0.1 \mathrm{M}$ phosphate buffered saline (PBS) buffer $2 \mathrm{X} 10 \mathrm{~min}$. The block was placed in $1 \% \mathrm{OsO}_{4}$ at $4^{\circ} \mathrm{C}$ for $1 \mathrm{~h}$, washed in $0.1 \mathrm{M}$ PBS ( $\mathrm{pH}$ 7.4) buffer overnight, rinsed in $\mathrm{ddH}_{2} \mathrm{O}$, dehydrated in a graded ethanol series and polymerized in embedding solution. The small block was cut into semi-thin sections using an LKB-V ultramicrotome until the point of interest was reached and then ultra-thin sections were cut. These sections were double stained with uranyl acetate and lead citrate and examined with a transmission electron microscope (TEM) (JEM-1200EX II, JEOL, Tokyo, Japan).

Table II. Antibodies used for immunohistochemical staining.

\begin{tabular}{llcl}
\hline Antibody & Catalog no. & Antigen retrieval & Manufacturer \\
\hline$\alpha$-smooth muscle actin & ZM-0003 & No & Zhongshan (Beijing, China) \\
Estrogen receptor & M3634 & Yes & Dako (Carpinteria, CA, USA) \\
Integrin $\beta 1$ & Sc-9970 & Yes & Dako (Carpinteria, CA, USA) \\
E-cadherin & MAB-0589 & Yes & Maxin (Fujian, China) \\
Focal adhesion kinase & bs-1340R & Yes & Biosan (Beijing, China) \\
Talin & sc-81805 & Yes & Santa Cruz (Santa Cruz, CA, USA) \\
Vinculin & sc-59803 & Yes & Santa Cruz (Santa Cruz, CA, USA) \\
\hline
\end{tabular}




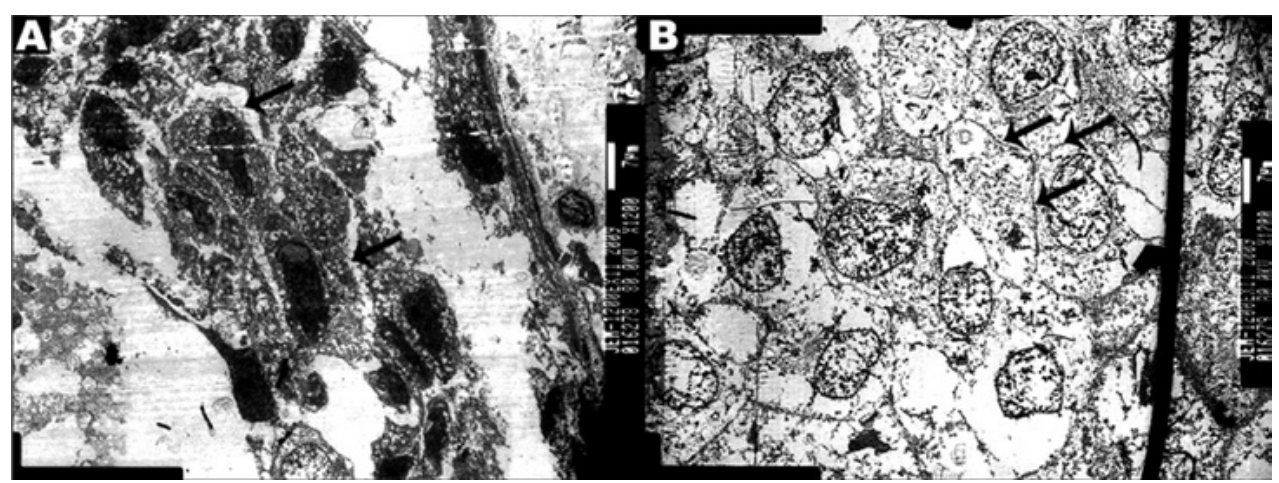

Figure 1. Appearance of cells overlying an FMCLD in DCIS and adjacent normal tumor cells under an electronic microscope. (A) Gaps are noted between cells overlying an FMCLD (arrows). The cells present elongated nuclei and stellate cell bodies, and the electronic density of nuclei and cell bodies is high. (B) The luminal cell counterpart has tight junctions (arrows), and the electronic density is low in nuclei and cell bodies.

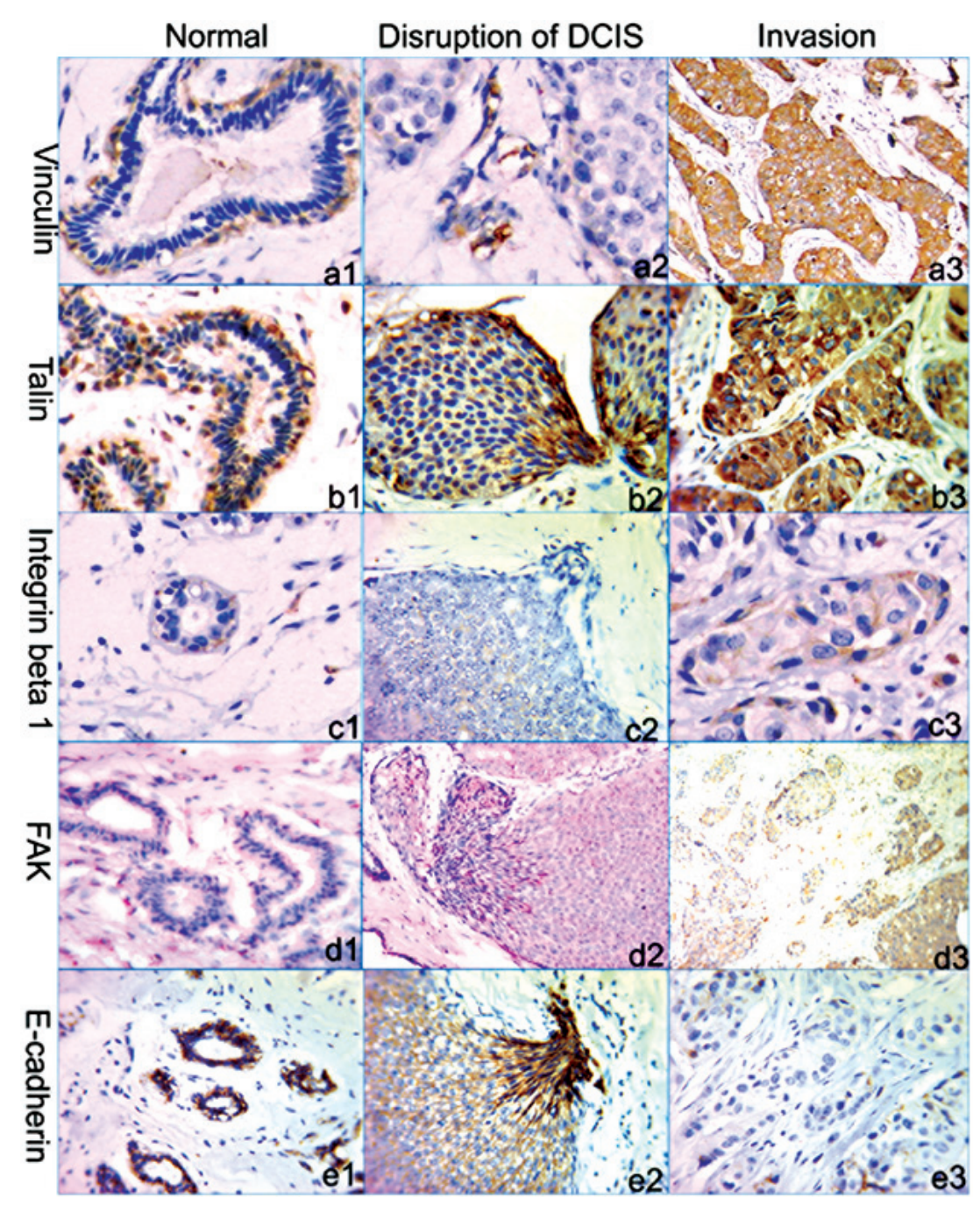

Figure 2. Immunohistochemical staining of breast cancer. Human breast tissue sections were immunostained for vinculin, talin, integrin $\beta 1$, FAK and E-cadherin (brown). All sections were stained with chromogen DAB, with the exception of FAK d1 and $\mathrm{d} 2$ stained with chromogen AP red. The left column shows the staining of normal mammary ducts, the middle column shows the staining of FMCLDs on DCIS and the right column shows the staining of an invasive tumor.

\section{Results}

Ultrastructural characteristics. Two FFPE blocks of ER-positive cases with FMCLD lesions were transformed to ultra-thin sections. Four FMCLD regions on the tissue blocks were carved out and also cut into ultrathin sections. The results of TEM showed that the tumor cells overlying FMCLDs had darkly stained nuclei and cytoplasm, with elongated nuclei 


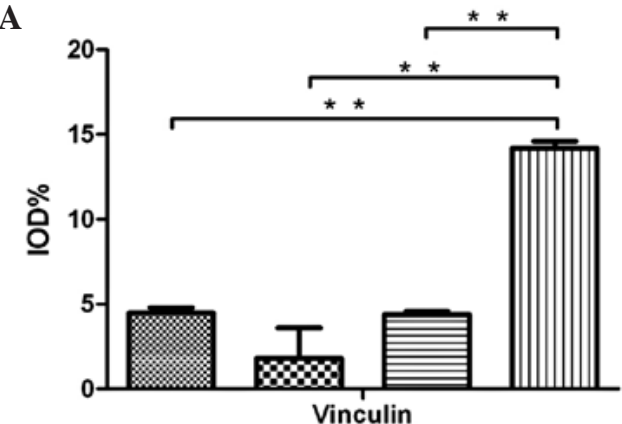

C

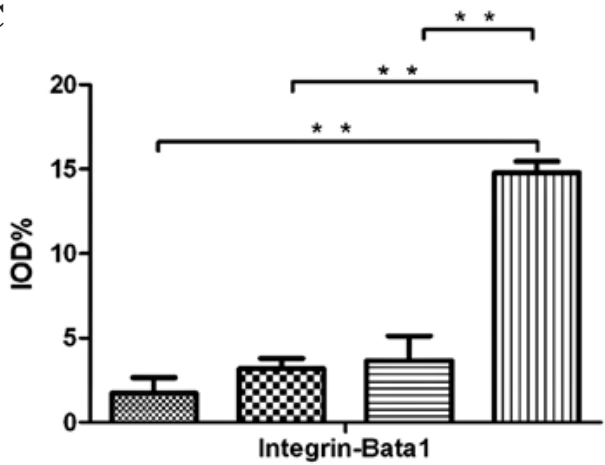

Normal

$\infty$ DCIS
B

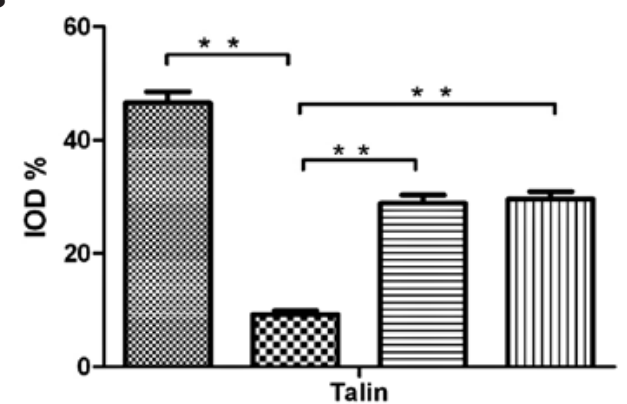

D

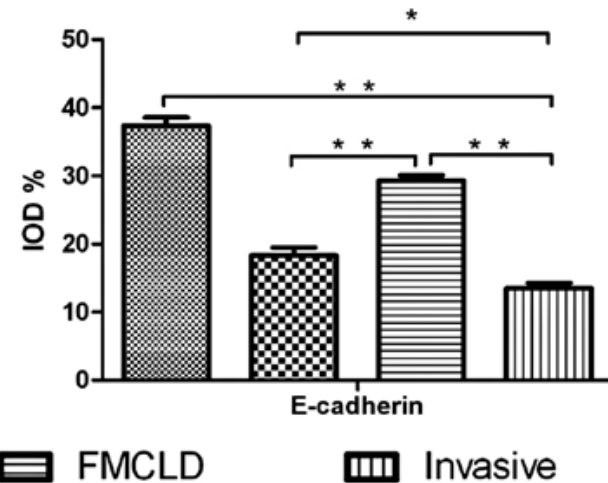

Figure 3. The average density of immunostaining (integrated optical density, IOD value). The IOD values of (A) a normal duct, (B) pure DCIS, (C) stained cells overlying an FMCLD and (D) a stained invasive tumor. Data are obtained from three lesions in each case and presented as the mean \pm standard error (SE). Asterisks indicate significant differences $\left({ }^{*} \mathrm{P}<0.05 ;{ }^{* *} \mathrm{P}<0.01\right)$ between the indicated cases calculated by one-way ANOVA test with the Holm-Sidak method.

and stellate cell bodies. The cells were separated from each other by thin gaps, 5-30 $\mu \mathrm{m}$ wide (Fig. 1A). Compared to these cells, the adjacent tumor cells within the associated duct were rounded, with weakly stained nuclei and cytoplasm, with high electronic density at the regions of membrane contact. This demonstrated that the membrane junction was tight between in situ tumor cells (Fig. 1B).

Expression of vinculin, talin, integrin $\beta 1, F A K$ and E-cadherin . Double immunohistochemical staining was performed on all 17 ER-positive cases for $\alpha$-SMA and ER to locate FMCLD lesions. Seven cases harbored FMCLD lesions and 61 FMCLD lesions were identified. Immunohistochemical staining for vinculin, talin, integrin $\beta 1$, FAK and E-cadherin was performed on these seven cases. The staining results showed that vinculin was strongly expressed (+++) in the cytoplasm of ME cells of all normal mammary ducts, while not expressed in luminal cells. The same result was also observed for $\alpha$-SMA staining. Of 61 ducts with FMCLD lesions in DCIS cases, vinculin was moderately $(++)$ or weakly $(+)$ expressed in ME cells, and not expressed in luminal tumor cells. The tumor cells overlying FMCLDs of 11 ducts (18\%) had very weak vinculin expression (+). Compared to the expression in normal ducts and DCIS lesions, in invasive lesions vinculin expression was elevated (++; Figs. 2a1, a2, a3 and 3A).

In normal ducts, strong talin expression (+++) was detected in the cytoplasm of both ME cells and parts of glandular epithelial cells. In DCIS lesions with FMCLDs, the cells overlying the disruptions were strongly talin-positive (+++), in sharp contrast to adjacent counterparts within the same ducts. Talin was also strongly expressed $(+++)$ in invasive lesions (Figs. 2b1, b2, b3 and 3B).

Integrin $\beta 1$ was detected in the cytoplasm of ME and luminal epithelial cells of normal ducts. In DCIS lesions, integrin $\beta 1$ was detected only in a small number of cells, and its staining was weak (+). No distinct difference in integrin $\beta 1$ expression was observed between the tumor cells overlying FMCLDs and their adjacent counterparts within the same duct. In invasive lesions, a relatively high expression (+) of integrin $\beta 1$ was detected, while only a portion of cells were stained (Figs. 2c1, c2, c3 and 3C).

FAK staining was weak $(+)$ in normal ducts and a higher level of expression (++) was detected in DCIS lesions of all cases. In the tumor cell clusters overlying FMCLDs, FAK was a bit more highly expressed (+++) than in the adjacent tumor cells within the same duct. FAK expression was weak (+) in invasive lesions and lower than in DCIS (Fig. 2d1, d2 and d3).

Strong E-cadherin expression $(+++)$ was detected in the plasma membranes of both ME and epithelial cells in normal ducts. Altered expression was detected in the cells overlying FMCLDs, where E-cadherin was strongly expressed (+++) in both the plasma membrane and cytoplasm, but only expressed in the membranes of adjacent tumor cells within the same duct. In invasive lesions, E-cadherin expression was only detected in a small number of tumor cells and the staining was very weak (+; Figs. 2e1, e2, e3 and 3D). 


\section{Discussion}

Previous studies have shown that a subset of cell clusters overlying FMCLDs of DCIS present many features similar to malignant tumors. Our present study further revealed that the cell clusters overlying FMCLDs were morphologically and immunohistochemically different from adjacent tumor cells within the corresponding duct: i) the cells emerging from FMCLDs had lost tight contacts with their neighbors and they had gained characteristics associated with cell motility; ii) the expression of talin and FAK in these cells were higher than that in adjacent cells, while vinculin and integrin $\beta 1 \mathrm{immu}-$ nostaining were infrequently detected; iii) by contrast, the expression of these molecules in the cells overlying FMCLDs was different from that in the epithelial cells of normal ducts and invasive tumor cells. These phenotypic characteristics are typically associated with the malignancy of tumor cells.

To migrate, the cell body must modify its shape and firmness to move through the surrounding tissue structures. Cells with motile capability generally present elongated nuclei and stellate cell bodies. Under TEM, we found that the cells overlying FMCLDs had polarized and elongated nuclei, and cell bodies with many protrusions. The cells had lost their tight junctions and there were gaps between them. These results imply that the tumor cell could fall off from the tumor cell cluster. All of these phenotypes are associated with cell motility, which have been described in many previous studies on cell motility and migration (18-20).

Cancer cell motility involves integrin signaling, focal adhesion formation and actomyosin-dependent contractility (21). Most breast tumor cells use chain migration or collective migration mechanisms (22-24). The molecular interactions that underlie changes in shape and regulate migration are mainly related to focal adhesion dynamics. There are several adhesion systems in normal mammary glands to maintain cell integrity. The main adhesion molecules are cadherins and integrins. To date, only a few studies have described the pathological characteristics of focal adhesion molecule expression in breast carcinoma in vivo. Glukhova et al (25) examined the expression of adhesion molecules in breast cancer, and reported that the expression of talin, vinculin and integrin was decreased in invasive breast tumors, compared to normal mammary ducts. Our study further confirmed their results. Also we demonstrated that the expression of talin, vinculin and integrin was higher in invasive lesions compared to that in DCIS. We also found that the expression of these molecules in cells overlying FMCLDs was different from that of luminal epithelial cells in the corresponding duct (Fig. 3). Therefore, altered expression in these cells may be a beginning event of tumor invasion.

Generally, E-cadherin maintains mammary gland epithelial cell junctions, whereas integrins are mainly distributed in basal layer cells to interact with the extracellular matrix. Previous studies provide evidence that many adhesion molecules, such as E-cadherin and integrins, are down-regulated in breast carcinoma and this down-regulation is associated with poor prognosis (26-28). However, interestingly, cells maintain a certain aggregation in invasive breast tumors. Our finding demonstrated that the expression levels of adhesion molecules, such as E-cadherin, vinculin and talin, were lower in DCIS ducts and invasive lesions than in normal ducts, while the expression levels of adhesion molecules in invasive lesions were higher than those in DCIS ducts. The likely explanation is that the loss of cell-cell junctions is a pre-requisite for cells to fall off the tumor core, which may allow them to spread into the stroma.

Although the tight junctions were reduced in the cells overlying FMCLDs, the cells also retained a degree of adhesion, which may contribute to their maintaining a certain integrity and ability to interact with the extracellular matrix, otherwise they would not be able to adapt to the stromal environment or migrate within it. When the ME cell layer is disrupted, tumor cells at the FMCLD are exposed to stroma and interact with extracellular matrix. This interaction perhaps induces the tumor cell to express elevated levels of adhesion molecules, and so the expression of talin, vinculin and FAK in the cells overlying the FMCLD is higher than that of the tumor cells within the corresponding duct.

In the progression from DCIS to invasive tumor, we found that the altered expression of talin, vinculin, integrin $\beta 1$ and FAK was not coordinated. The expression level of talin was markedly higher in the cells overlying the FMCLD than that in their counterparts of the same duct, while integrin and vinculin were weakly expressed (Fig. 3). Many previous studies confirm talin is required for cells to form new adhesions on fibronectin during cell spreading and migration (29-31). Since talin plays an important role in forming focal adhesions, a high talin expression in cells overlying FMCLDs implies that talin is of prime importance in initiating the invasion of the tumor cell through the stroma. However, details of the signaling pathways that regulate talin activity are only just beginning to emerge, and the structural basis for activation of the many ligand-binding sites in talin is not yet fully understood. The role of talin in the early stages of tumor invasion needs to be addressed.

Vinculin plays a crucial role in linking focal adhesions to actin-cytoskeleton. Generally, its expression is restricted in ME cells of the normal mammary duct. It is lowly expressed in the ME of DCIS and not expressed during the disruption of the ductal wall. Our results demonstrated that vinculin was very weakly or not expressed in the cells overlying FMCLDs, while highly expressed in invasive lesions. Compared to talin, vinculin expression was much weaker. Vinculin activation may lag behind that of talin in the early stages of invasive tumor development. Recent studies found that vinculin regulates cell-surface E-cadherin expression by binding to $\beta$-catenin (32-34). Our result confirmed this point; vinculin expression was increased in invasive tumors and that of E-cadherin gradually decreased as cells transformed from luminal cells of normal ducts to invasive tumor cells. However, altered E-cadherin expression in the cells reflects the complex molecular changes in the early stage of tumor invasion. By contrast, the expression of vinculin gradually increased as cells moved from overlying FMCLDs to invasive tumor cells in the stroma. This means that as E-cadherin-mediated cellcell adhesion is reduced, it is replaced by integrin-mediated cell-cell or cell-matrix adhesion and motility.

FAK is involved in integrin-mediated signaling which has a profound impact on cell proliferation, survival and migration. Previous studies (35-37) suggest that FAK overexpression is significantly associated with HER2 overexpression and 
Akt phosphorylation. Akt may be a downstream target of FAK-mediated signaling. Since Akt activation is associated with a worse prognosis in breast cancer, and HER2 overexpression is associated with tumor migration, FAK overexpression may play an important role in promoting tumor progression. Similar to talin, elevated FAK expression in DCIS and the cells overlying FMCLDs imply their importance in the early stage of invasive tumor development. However, the invasive lesion exhibited lower FAK immunostaining than DCIS. The reason remains unknown and further studies are required to address this issue.

Many reports have attempted to discover the mechanism by which DCIS develops into invasive tumor; the histological characteristics of DCIS and invasive tumors have been widely examined. However, the tumor cells overlying FMCLDs of DCIS have been rarely studied, perhaps because: i) as the disruption of the ME cell layer is usually very small, it is difficult to identify in clinical diagnostic histology by $\mathrm{H} \& \mathrm{E}$ staining and can only be detected by immunohistochemistry; ii) past studies have primarily focused on pure DCIS and invasive lesions; and iii) the frequency of these lesions in the clinical setting is low $(\sim 15 \%)$.

The development from breast DCIS to invasive tumor is a complex process. Our findings elucidated both the expression characteristics of molecular markers that are correlated with cell adhesion and motility, and the ultrastructural characteristics of the cells overlying FMCLDs. These findings may provide more detailed information about the origin of invasive tumor cells and facilitate early diagnosis of breast cancer.

Since the frequency of these lesions in the clinic is relatively low, there is still much work to do to investigate the characteristics of tumor cells overlying FMCLDs.

In summary, the present study provided additional morphological, ultrastructural and immunohistochemical data confirming that the cells overlying FMCLDs likely represent the specific precursor of invasive breast lesions. Our findings may facilitate the identification of specific targets for further molecular profiling in order to characterize this important cell population.

\section{Acknowledgements}

This study was supported in part by grant 2006CB910505 from the Ministry of Chinese Science and Technology Department to Dr Xi-Chen Zhang. The authors thank Medjaden Bioscience Ltd. for assisting in the preparation of this manuscript.

\section{References}

1. Beckmann MW, Niederacher D, Schnurch HG, Gusterson BA and Bender HG: Multistep carcinogenesis of breast cancer and tumour heterogeneity. J Mol Med 75: 429-439, 1997.

2. Schmitt FC: Multistep progression from an oestrogen-dependent growth towards an autonomous growth in breast carcinogenesis. Eur J Cancer 31 A: 2049-2052, 1995.

3. Clarke R, Brunner N, Katzenellenbogen BS, et al: Progression of human breast cancer cells from hormone-dependent to hormoneindependent growth both in vitro and in vivo. Proc Natl Acad Sci USA 86: 3649-3653, 1989.

4. Tsubura A, Shikata N, Inui $\mathrm{T}$, et al: Immunohistochemical localization of myoepithelial cells and basement membrane in normal, benign and malignant human breast lesions. Virchows Arch A Pathol Anat Histopathol 413: 133-139, 1988.
5. Jolicoeur F, Seemayer TA, Gabbiani G, et al: Multifocal, nascent, and invasive myoepithelial carcinoma (malignant myoepithelioma) of the breast: an immunohistochemical and ultrastructural study. Int J Surg Pathol 10: 281-291, 2002.

6. Slade MJ, Coope RC, Gomm JJ and Coombes RC: The human mammary gland basement membrane is integral to the polarity of luminal epithelial cells. Exp Cell Res 247: 267-278, 1999.

7. Miosge $\mathrm{N}$ : The ultrastructural composition of basement membranes in vivo. Histol Histopathol 16: 1239-1248, 2001.

8. Nerlich A: Morphology of basement membrane and associated matrix proteins in normal and pathological tissues. Veroff Pathol 145: 1-139, 1995 (In German).

9. Man YG, Tai L, Barner R, et al: Cell clusters overlying focally disrupted mammary myoepithelial cell layers and adjacent cells within the same duct display different immunohistochemical and genetic features: implications for tumor progression and invasion. Breast Cancer Res 5: R231-R241, 2003.

10. Yousefi M, Mattu R, Gao C and Man YG: Mammary ducts with and without focal myoepithelial cell layer disruptions show a different frequency of white blood cell infiltration and growth pattern: implications for tumor progression and invasion. Appl Immunohistochem Mol Morphol 13: 30-37, 2005.

11. Man YG, Zhang Y, Shen T, et al: cDNA expression profiling reveals elevated gene expression in cell clusters overlying focally disrupted myoepithelial cell layers: implications for breast tumor invasion. Breast Cancer Res Treat 89: 199-208, 2005.

12. Man YG, Zhao CQ and Wang J: Breast tumor cell clusters and their budding derivatives show different immunohistochemical profiles during stromal invasion: implications for hormonal and drug therapies. Cancer Ther 4: 193-204, 2006.

13. Zhang $\mathrm{X}$, Hashemi SS, Yousefi M, et al: Aberrant c-erbB2 expression in cell clusters overlying focally disrupted breast myoepithelial cell layers: a trigger or sign for emergence of more aggressive cell clones? Int J Biol Sci 4: 259-269, 2008.

14. Banyard $\mathbf{J}$ and Zetter BR: The role of cell motility in prostate cancer. Cancer Metastasis Rev 17: 449-458, 1998.

15. Yam JW, Tse EY and Ng IO: Role and significance of focal adhesion proteins in hepatocellular carcinoma. J Gastroenterol Hepatol 24: 520-530, 2009.

16. Albiges-Rizo C, Destaing O, Fourcade B, Planus E and Block MR: Actin machinery and mechanosensitivity in invadopodia, podosomes and focal adhesions. J Cell Sci 122: 3037-3049, 2009.

17. Man YG and Tavassoli FA: A simple epitope retrieval method without the use of microwave oven or enzyme digestion. Appl Immunohistochem 4: 139-141, 1996.

18. Friedl P and Brocker EB: The biology of cell locomotion within three-dimensional extracellular matrix. Cell Mol Life Sci 57: 41-64, 2000.

19. Mseka T, Coughlin M and Cramer LP: Graded actin filament polarity is the organization of oriented actomyosin II filament bundles required for fibroblast polarization. Cell Motil Cytoskeleton 66: 743-753, 2009.

20. Popow-Wozniak A, Nowak D and Malicka-Blaszkiewicz M: [Types of tumor cells movement]. Postepy Biochem 55: 113-120, 2009.

21. Friedl $\mathrm{P}$ and Wolf $\mathrm{K}$ : Tumour-cell invasion and migration: diversity and escape mechanisms. Nat Rev Cancer 3: 362-374, 2003.

22. Pitts WC, Rojas VA, Gaffey MJ, et al: Carcinomas with metaplasia and sarcomas of the breast. Am J Clin Pathol 95: 623-632, 1991.

23. Klinowska TC, Soriano JV, Edwards GM, et al: Laminin and betal integrins are crucial for normal mammary gland development in the mouse. Dev Biol 215: 13-32, 1999.

24. Simian M, Hirai Y, Navre M, Werb Z, Lochter A and Bissell MJ: The interplay of matrix metalloproteinases, morphogens and growth factors is necessary for branching of mammary epithelial cells. Development 128: 3117-3131, 2001.

25. Glukhova M, Koteliansky V, Sastre X and Thiery JP: Adhesion systems in normal breast and in invasive breast carcinoma. Am J Pathol 146: 706-716, 1995.

26. Coopman PJ, Thomas DM, Gehlsen KR and Mueller SC: Integrin alpha 3 beta 1 participates in the phagocytosis of extracellular matrix molecules by human breast cancer cells. Mol Biol Cell 7: 1789-1804, 1996.

27. Teuliere J, Faraldo MM, Deugnier MA, et al: Targeted activation of beta-catenin signaling in basal mammary epithelial cells affects mammary development and leads to hyperplasia. Development 132: 267-277, 2005. 
28. Mierke CT, Kollmannsberger P, Zitterbart DP, et al: Vinculin facilitates cell invasion into three-dimensional collagen matrices. J Biol Chem 285: 13121-13130, 2010.

29. Tanentzapf G, Martin-Bermudo MD, Hicks MS and Brown $\mathrm{NH}$ : Multiple factors contribute to integrin-talin interactions in vivo. J Cell Sci 119: 1632-1644, 2006.

30. Critchley DR and Gingras AR: Talin at a glance. J Cell Sci 121 1345-1347, 2008.

31. Beckerle MC and Yeh RK: Talin: role at sites of cell-substratum adhesion. Cell Motil Cytoskeleton 16: 7-13, 1990.

32. Berx G and van Roy F: The E-cadherin/catenin complex: an important gatekeeper in breast cancer tumorigenesis and malignant progression. Breast Cancer Res 3: 289-293, 2001.

33. Le Duc Q, Shi Q, Blonk I, et al: Vinculin potentiates E-cadherin mechanosensing and is recruited to actin-anchored sites within adherens junctions in a myosin II-dependent manner. J Cell Biol 189: 1107-1115, 2010
34. Sawada K, Mitra AK, Radjabi AR, et al: Loss of E-cadherin promotes ovarian cancer metastasis via alpha 5-integrin, which is a therapeutic target. Cancer Res 68: 2329-2339, 2008.

35. Schmitz KJ, Grabellus F, Callies R, et al: High expression of focal adhesion kinase (p125FAK) in node-negative breast cancer is related to overexpression of HER-2/neu and activated Akt kinase but does not predict outcome. Breast Cancer Res 7: R194R203, 2005.

36. Ren XD, Kiosses WB, Sieg DJ, Otey CA, Schlaepfer DD and Schwartz MA: Focal adhesion kinase suppresses Rho activity to promote focal adhesion turnover. J Cell Sci 113: 3673-3678, 2000.

37. Lark AL, Livasy CA, Dressler L, et al: High focal adhesion kinase expression in invasive breast carcinomas is associated with an aggressive phenotype. Mod Pathol 18: 1289-1294, 2005. 